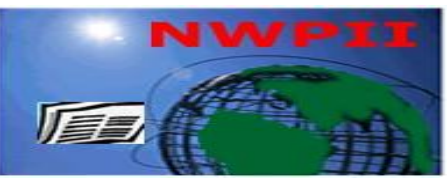

American Journal of Biomedical Sciences

ISSN: 1937-9080

nwpii.com/ajbms

\title{
A Simple Parallel Plate Flow Chamber to Study Effects of Shear Stress on Endothelial Cells
}

\begin{abstract}
Harmanpreet Kaur ${ }^{1}$, Rupp Carriveau ${ }^{2}$ and Bulent Mutus ${ }^{1 *}$
${ }^{1}$ Departments of Chemistry and Biochemistry, University of Windsor, Windsor, Ontario, Canada.

${ }^{2}$ Civil and Environmental Engineering, University of Windsor, Windsor, Ontario, Canada.

*Corresponding author:

Bulent Mutus

Department of Chemistry and Biochemistry

University of Windsor

Windsor, Ontario

Canada, N9B 3P4

Phone: 519-253-3000 ext 3533

Email: mutusb@uwindsor.ca

Received: 18 September 2011; | Revised: 10 November 2011; | Accepted: 16 November 2011

Abstract

In the present study, we report the construction of a parallel plate flow chamber for studying the effects of shear stress in endothelial cells. This flow chamber is inexpensive and simple to manufacture and use. Moreover, it minimizes the number of cells and solution volumes to be used. Here, bovine aortic endothelial cells (BAECs) were either maintained in static condition or exposed to laminar shear stress ( $4 \& 8 \mathrm{dyne} / \mathrm{cm}^{2}$ ) for $16 \mathrm{~h}$. Shear stress markedly and significantly up-regulated nitric oxide production in endothelial cells as compared to the static cells. An increase in caveolin-1 expression and eNOS phosphorylation at Ser1177 was also observed in BAECs upon exposure to shear stress. This study demonstrates that our newly designed parallel plate flow chamber is well suited for the in vitro system for the examination of the effects of shear stress on endothelial cells.
\end{abstract}

Keywords: Shear stress, parallel flow chamber, laminar flow, endothelial cells.

\section{Introduction}

Endothelial cells are exposed to various mechanical stimuli such as shear stress, hydrostatic pressure and cyclic stretch due to vessel deformation. These stimuli alter endothelial cell morphology, initiate cytoskeletal changes and activate various signalling pathways. The nature and magnitude of these hemodynamic forces has an important role in maintaining vascular homeostasis.

Laminar shear stress is one of the most potent endothelial stimulators, which is very critical for normal vascular functioning. It plays an important role against inflammatory activation and apoptosis $[1,2]$. Disturbed shear stress has been implicated 
in the pathogenesis of various diseases including atherosclerosis and cardiovascular diseases [3].

In the past few years, physiological and pathological shear stress has been studied extensively to understand the development and progression of vascular diseases. To understand the effects of these hemodynamic forces in vitro, engineered devices are required that can maintain the endothelial cells in a controlled in vivo like environment. The fluid flow must be predictable in these devices so that shear stress over endothelial cells can be properly controlled.

Many in vitro flow systems have been developed to study endothelial cells under shear stress. These flow devices require a large population of endothelial cells which needs more chemicals and reagents. Recently, many studies have developed microfluidic flow chambers. Some of these designs have multiple microchannels to study different cell types and shear rates at the same time [4]. A major drawback of such flow devices is cross contamination between different channels. Some of these flow devices and microfluidic devices have been custom laboratory prototypes, while others are commercially available.

In the present study, we describe the construction and testing of a simple and robust parallel plate flow chamber. The aim of this study was to develop an in vitro model using a parallelplate flow chamber to simulate in vivo fluid shear stresses on endothelial cells exposed to dynamic fluid flow in their physiological environment. A Computational Fluid Dynamics (CFD) template was developed via FLUENT ${ }^{\mathrm{TM}}$ for simulation and prediction of flow induced shear rates on the endothelium, while an in vitro model was used to investigate the subsequent cellular biological responses.

The flow chamber was validated by determining changes in nitric oxide production and caveolin-1 expression in bovine endothelial cells exposed to shear stress.

\section{Materials and Methods}

\subsection{Cell culture}

Bovine aortic endothelial cells (BAECs) were purchased from Coriell Institute for Medical
Research USA. The cells were cultured in DMEM F-12 media (Sigma, Canada) containing $10 \%$ fetal calf serum (FCS, Gibco Canada) and penicillin-streptomycin (Gibco, Canada), at $37^{\circ} \mathrm{C}$ in a humidified atmosphere of $95 \%$ air and $5 \%$ $\mathrm{CO}_{2}$.

\subsection{Parallel plate flow chamber configuration}

The flow apparatus used in this study is a parallel plate flow chamber with a closed loop system. The configuration of the chamber is shown in Fig. 1.

\subsection{Viscosity measurement}

In order to use the velocity gradient templates as shear stress templates, the viscosity of the media at $37^{\circ} \mathrm{C}$ was required. A viscometer (Canon Instruments, Model 2085, PA, USA) and viscosity bath, set to $37^{\circ} \mathrm{C}$, was used. As per the procedure, the media was injected into the viscometer and allowed to reach equilibrium conditions before being suctioned to the top of the viscometer and then released while the time it takes the fluid to flow through a defined section was measured. Using equipment specific correlations, the viscosity was calculated based on the time taken to pass through a defined section. The latter was repeated five times and an average viscosity was calculated and used in the shear stress calculations. The specific gravity of the media was determined by using a calibrated hydrometer. The average viscosity was $0.753 \mathrm{cP}(0.00753 \mathrm{~Pa} \cdot \mathrm{s})$.

\subsection{Calculation of shear stress}

Shear stress was calculated according to the following formula:

$$
=\frac{d u}{d z}
$$

Where $\mu$ is the viscosity of the media and $\mathrm{du} / \mathrm{dz}$ is the velocity gradient of the flow. From the templates generated in FLUENT ${ }^{\mathrm{TM}}$ the values of the velocity gradients at various regions on the plate are known. Since the viscosity of the media has been measured, the shear stress can be directly calculated and an average shear stress for each region on the template can be defined. The shear stress values used in our investigations ranged from 0.05 to $1.47 \mathrm{~Pa}$, which is within the 
physiological range for arteries $(\sim 0.2 \mathrm{~Pa}-6 \mathrm{~Pa})$ and veins $(\sim 0.01 \mathrm{~Pa}-0.6 \mathrm{~Pa})$.

\subsection{Shear stress studies}

Bovine endothelial cells were grown on coverslips in $35 \mathrm{~mm}$ cell culture plates. The cells were exposed to shear stress using peristaltic pump (Reglo Digital MS 4/6, model ISM 833, Ismatec). PTFE tubing (Scientific Products \& Equipment, Canada) was used to link the pump and the flow chamber. Shear stress experiments were performed for 16 hours in complete media in $5 \% \mathrm{CO}_{2}$ incubator at $37^{\circ} \mathrm{C}$.
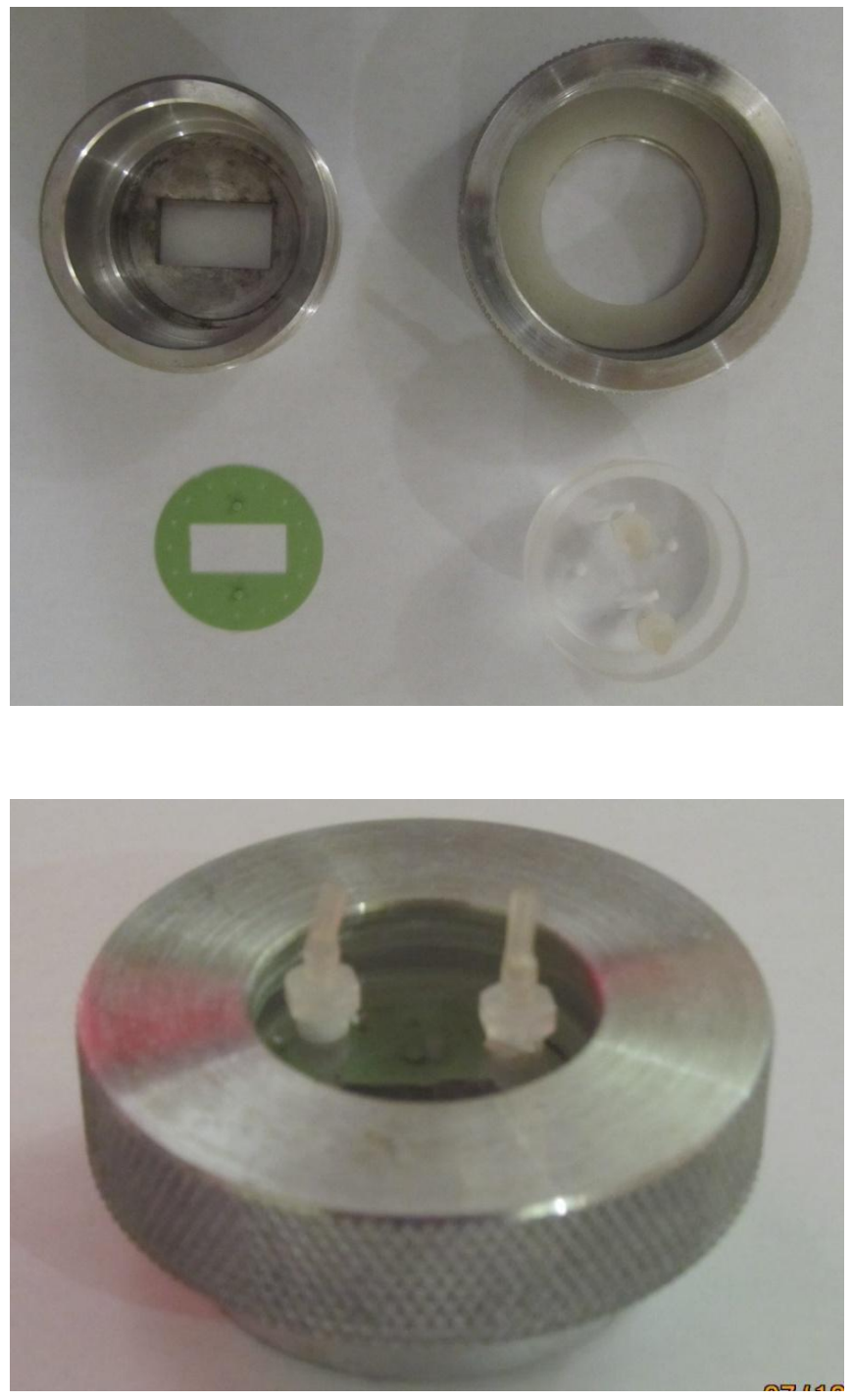

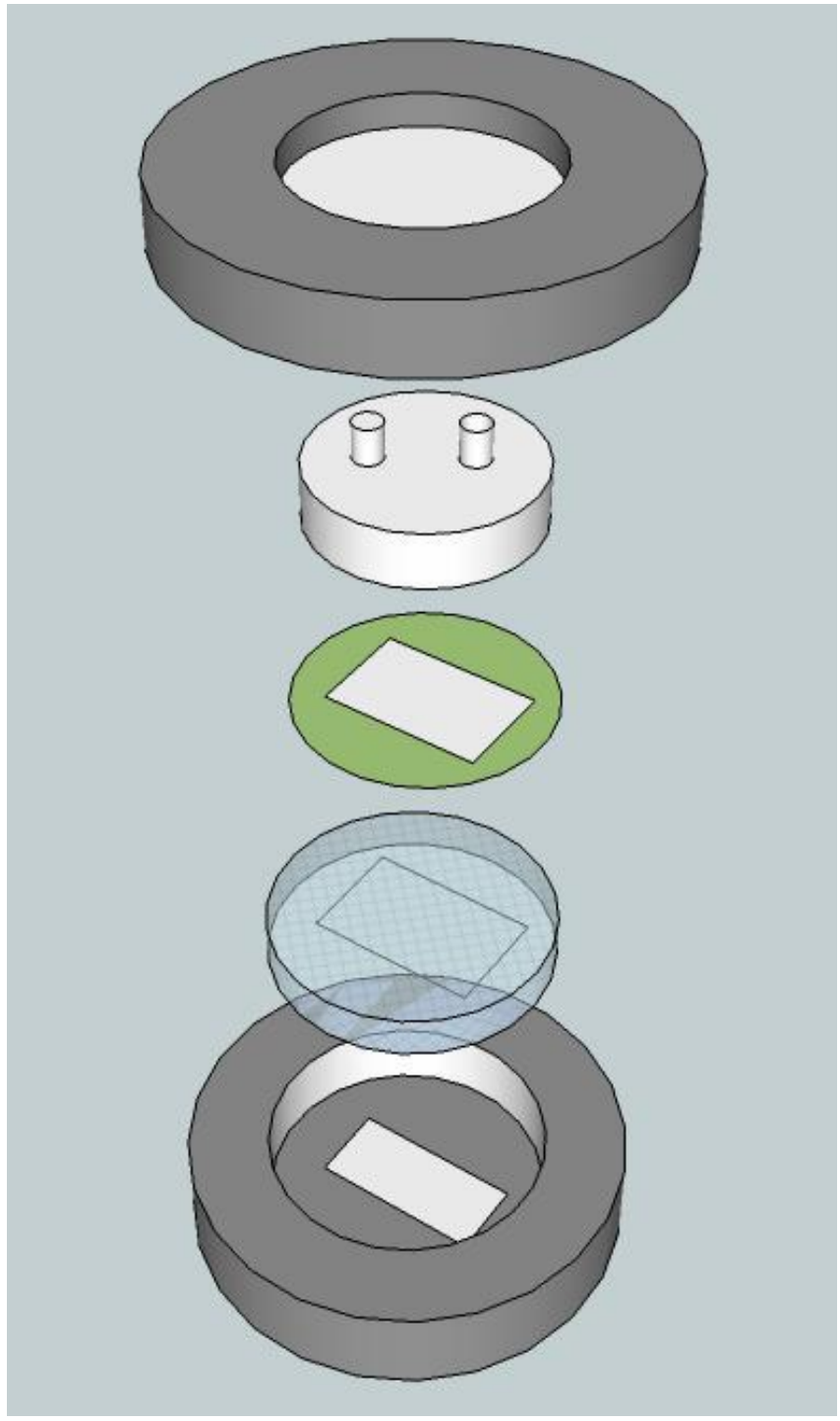

Figure 1. Parallel plate flow chamber

\subsection{Measurement of NO production}

To determine changes in NO production, endothelial cell monolayers were incubated with cell culture medium containing $5 \mu \mathrm{M}$, 4,5diaminofluorescein diacetate (DAF-DA, Invitrogen Canada) at $37^{\circ} \mathrm{C}$ in a $95 \%$ air $/ 5 \% \mathrm{CO}_{2}$ incubator for $30 \mathrm{~min}$. Cells were then washed $3 \mathrm{X}$ with phosphate buffered saline (PBS) and mounted on the stage of an Axiovert 200 inverted fluorescence microscope. DAF-DA fluorescence was monitored using excitation and emission wavelengths of 485 and $538 \mathrm{~nm}$, respectively.

BAECs were grown under shear stress and static cells were used as control. After incubation for 24 hours, media containing $100 \mu \mathrm{M}$ of 
acetylcholine (Sigma, Canada) was injected into the flow chamber. The cells were incubated again and after $1 \mathrm{hr}, 200 \mu \mathrm{L}$ of media was withdrawn with a Hamilton gastight syringe and directly injected into the purge vessel of a Sievers Nitric Oxide Analyzer (Model 280i) which contained acetic acid and sodium iodide.

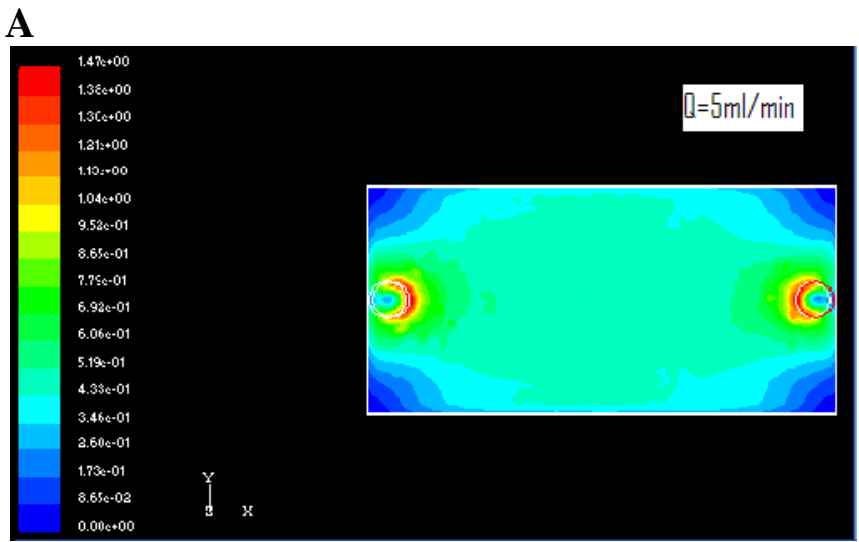

B

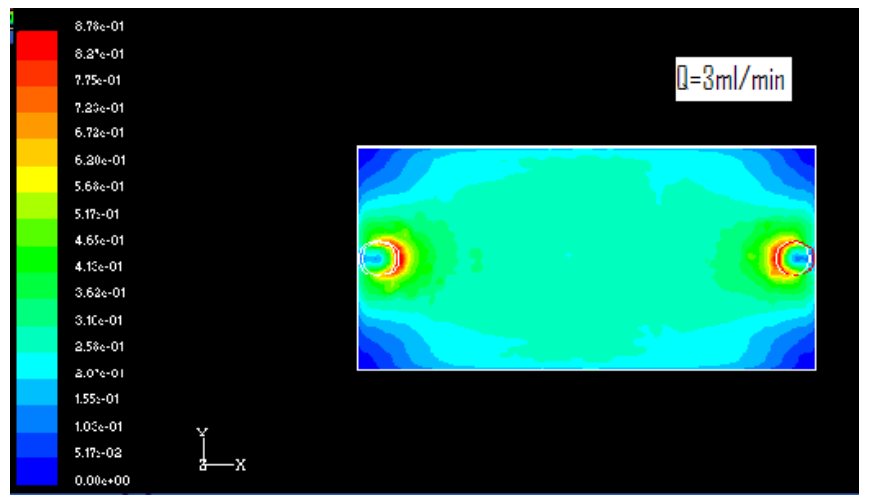

Figure 2. Velocity gradient template

\subsection{Immunofluorescence}

BAEC cells were grown on coverslips in $35 \mathrm{~mm}$ cell culture dishes. The cells were grown under shear stress for 16 hours and static cells were used as control. After incubation, the cells were washed with PBS and fixed in $3 \%$ paraformaldehyde for $10 \mathrm{~min}$. The cells were then permeabilized with $0.025 \%$ Triton $\mathrm{X}$ for $10 \mathrm{~min}$. Rabbit polyclonal antibody against Ser 1177 phospho eNOS (1:200 dilution; Cell Signal) and anti-mouse caveolin-1 (1:100) were used as primary antibodies. Alexa Fluor 488 -labelled goat anti-rabbit IgG and Alexa Fluor 568 conjugated anti-mouse $\operatorname{IgG}$ were used as a secondary antibodies (1:500 dilution; Molecular Probes,
Canada). Cells were also stained with either PI or DAPI for the localization of nuclei. Preparations were mounted in fluoromount $G$ (Southern Biotech, Canada) and examined by fluorescence microscopy.

\subsection{Statistical analysis}

Data is expressed as an average of all trials and statistical analysis was done by student's $t$ test using Microsoft Excel. The quantification of the fluorescence intensity was done using Image $\mathbf{J}$ software. The error bars in the figures represent standard deviation.

\section{Results and Discussion}

\subsection{Flow chamber}

Parallel plate flow chambers have been used for years in the study of hemodynamics and cellular mechanotransduction [5-9]. The majority of chambers documented in the literature were developed for specific research applications and were not mass-produced for commercial use. The bulk of chambers presently available off the shelf can cost upwards of $\$ 500$ for a pump-ready apparatus. They often require the use of a vacuum line or pump and delicate gaskets to maintain chamber seals and prevent leaks.

The parallel plate flow chamber described herein was designed to minimize solution volumes, to be operationally robust and precise, simple to set up, and inexpensive. Also critical to the design was the ability of the chamber to be mounted on a microscope stage to facilitate visualization experiments. The chamber pictured in Fig. 1 is $64 \mathrm{~mm}$ in diameter and $28 \mathrm{~mm}$ in height. It was machined out of surplus aluminum and acrylic stock. The unit consists of three principal elements, an inner acrylic "puck" that forms the chamber, an interchangeable gasket that creates the channel geometry, and an aluminum clamping fixture that holds it all together. A special low cost thermoset plastic has been adapted for use as a chamber gasket. A simple series of die punches provide a variety of channel geometries. The unit can also accept commercially available gaskets like those produced by Glycotech (Rockville Maryland). Channel depth is set by the clamping fixture and the thickness of 
gasket utilized. The two halves of the clamping fixture thread together to compress the gasket to provide the chamber seal. To ensure proper channel depth, two interchangeable locator pins prevent the fixture from being over tightened.

Inlet and outlet ports were machined into the top of the acrylic chamber. Each is threaded to accept standard commercially available fittings. The ports are chamfered with a smooth Gaussian shaped profile to minimize flow disturbances during flow entrance and exit.

\subsection{Computational fluid dynamics}

To simulate the flow in the chamber and estimate the shear stress for the testing program, a computational model of the flow channel was developed. The commercial finite volume solver, FLUENT $^{\mathrm{TM}} 6.1$ was utilized for this study. Free outflow and mass flow inlet boundary conditions were assumed at the outlet and inlet of the channel respectively in the steady, laminar simulation. The inlet/outlet ports and flow channel [19 mm long X $5 \mathrm{~mm}$ wide $X 0.25 \mathrm{~mm}$ deep] was discretized by tetrahedral elements. Three mesh densities were tested, 83,000, 196,000, and 454,000 elements. Values of the flow velocity assessed at 5 locations across the domain changed by no more than $2 \%$ when the mesh density increased from 196,000 to 454,000. Thus the medium mesh density was chosen for the test runs.

\subsection{Validation}

The reorganization of the endothelial cell morphology is one of the earliest responses in the endothelial cells that are exposed to fluid shear stress. Previous in vivo and in vitro studies have shown that the endothelial cells elongate and align parallel to the direction of flow $[10,11]$. In the present study, we first examined the morphological changes of endothelial cells associated with exposure to physiologically relevant shear stress using this flow chamber. Bovine aortic endothelial cells were either maintained in static condition or exposed to laminar flow at a shear stress of $4 \mathrm{dyne} / \mathrm{cm}^{2}$ for 16 h. As shown in Figure 3, fluid shear stress induced morphological changes such as cell elongation and alignment with the direction of flow. In contrast, there is no significant cell orientation in cells under static conditions. These results are consistent with those reported previously by other groups [12-14].

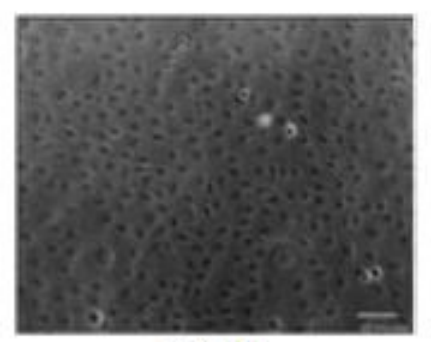

Static

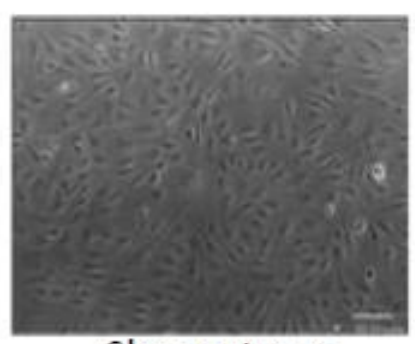

Shear stress

(4 dyne $/ \mathrm{cm}^{2}$ )
Figure 3. Effect of shear stress on morphology of bovine endothelial cells. Bovine aortic endothelial cells were maintained either under static conditions or under shear stress $\left(4 \mathrm{dyne} / \mathrm{cm}^{2}\right)$. After incubation for 16 hours, the morphological changes were visualized using 20X objective of a Zeiss Axiovert 200 microscope.

In order to validate whether this flow chamber can be employed for studies in the areas of biomedical research, the effect of fluid shear stress on nitric oxide production in endothelial cells was examined. Shear stress is one of the most important physiological stimuli which modulate many physiological and pathological processes associated with endothelium, such as inflammatory response, vasodilation and endothelial cell proliferation [15]. It acts as a potent stimulus for endothelium-dependent NO production and vasodilation [16].

In the present study, nitric oxide production in bovine aortic endothelial cells was determined by a membrane-permeable fluorescent probe 4, 5diaminofluorescein diacetate (DAF-2 DA). An important step in the validation process was to determine whether our flow chamber was able to discern different shear rates. BAECs were exposed to two different shear rates ( $4 \& 8$ dyne $/ \mathrm{cm}^{2}$ ). A qualitative examination of the data revealed that NOx production increased by 7 fold as compared to the static cells when the endothelial cells were exposed to a shear rate of $8 \mathrm{dyne} / \mathrm{cm}^{2}$ (Fig. $4 \mathrm{~A}$, B). A significant upregulation ( 2.5 fold $)$ of authentic NO (as determined with NO analyzer) was also observed in endothelial cells which were exposed to laminar flow at a shear stress of 4 
dyne $/ \mathrm{cm}^{2}$ for 16 hours, compared with those maintained in static condition (Fig. 4C). These findings are in agreement with numerous previous studies which demonstrated nitric oxide upregulation in response of laminar shear stress $[17,18]$.

\section{Figure 4}

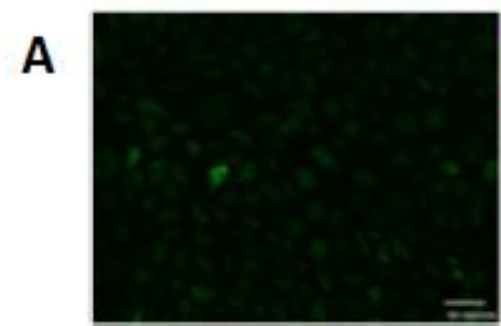

Static

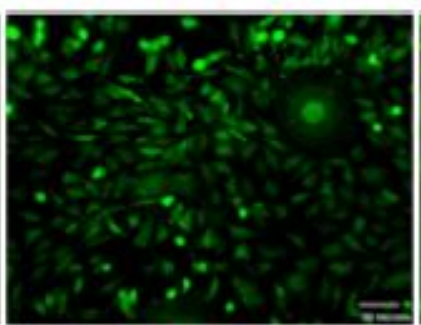

4 dyne $/ \mathrm{cm}^{2}$

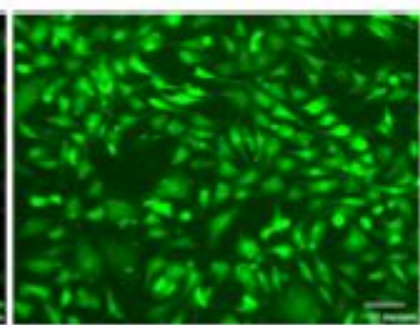

8 dyne $/ \mathrm{cm}^{2}$
B
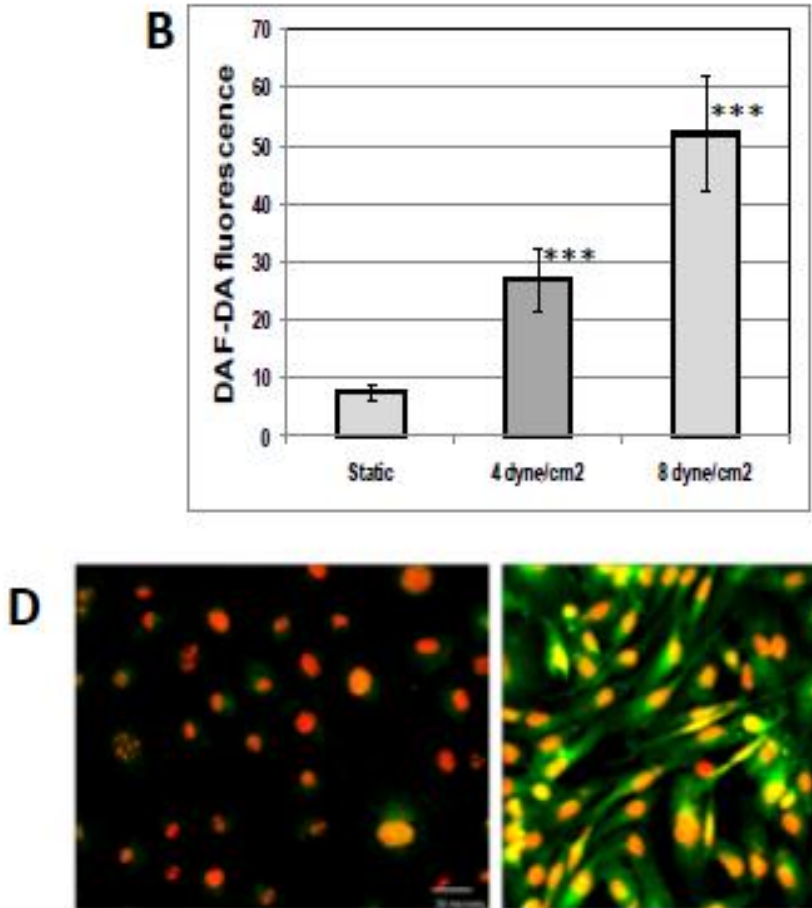

Static

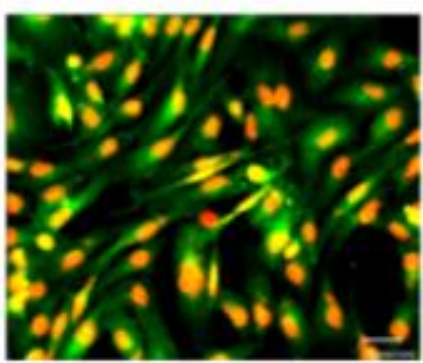

4 dyne $/ \mathrm{cm}^{2}$
C
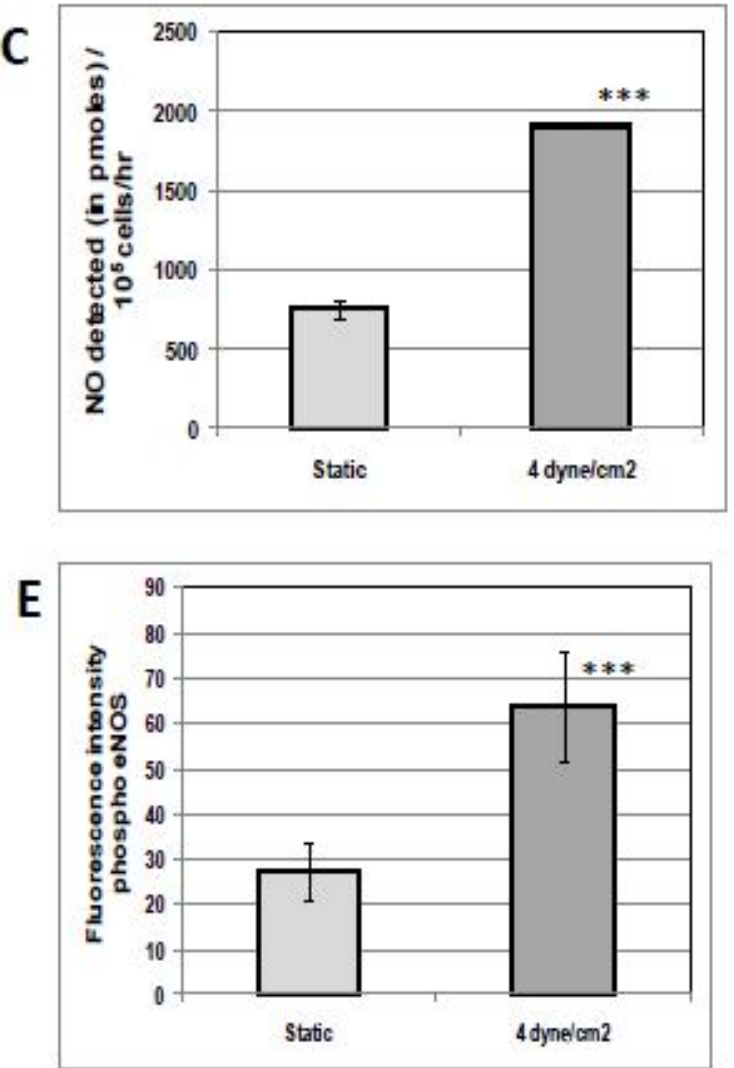

Figure 4. Nitric oxide production and eNOS phosphorylation in response to shear stress. (A) The bovine endothelial cells grown under static conditions and shear stress for 16 hours were incubated with $5 \mu$ M DAF-DA for 30 min. The cells were washed with PBS and viewed under Zeiss Axiovert fluorescence microscope using 20X objective. (B) The graph represents average of DAF-DA fluorescence taken from 4 independent experiments $( \pm \mathrm{SD})$. $* * * \mathrm{P}<$ 0.001 (C) Total nitrite was measured in control cells and cells under shear stress using NO analyzer. The bovine endothelial cells were grown under shear stress for 16 hours after which the cells were stimulated with acetylcholine for 1 hour and total nitrite was measured. The data shown is average of 3 independent experiments $( \pm$ SD). (D) The cells maintained under static conditions and exposed to shear stress for 16 hours were fixed with paraformaldehyde and incubated with anti-rabbit Ser1177 phospho eNOS antibody. Alexa Fluor 488 conjugated anti-rabbit IgG was used as secondary antibody and the nuclei were stained with propidum iodide (PI). The slides were viewed under oil immersion 40X objective. (E) Average gray values (immunofluorescence intensity) of phospho eNOS levels in static and shear stress exposed cells. The values (average \pm SD) were calculated from at least 10 different microscopic fields in each slide $(\mathrm{n}=3)$. $* * * \mathrm{P}<0.001$. 
Previous studies have also shown that increase in shear stress increases eNOS activity by phosphorylation of eNOS at serine 1177 [19, 20]. Phosphorylation of eNOS was determined by indirect immunofluorescence and the results of our study also illustrated increase in phosphorylation of eNOS at Ser 1177 by 2.3 fold (Fig.4 D, E). Caveolae are small cell surface plasma membrane invaginations, which have important role in cell signaling and cholesterol homeostasis. Cholesterol binding protein caveolin-1 is the main marker of caveolae. Caveolin-1 could mediate the transfer of newly synthesized cholesterol from the ER to the plasma membrane [21]. The changes in caveolin-1 expression and distribution were determined by indirect immunofluorescence. The cells exposed to shear stress showed a 2.7 fold increase in caveolin-1 expression as compared to static cells. In static cells, caveolin-1 is present mostly in the plasma membrane, whereas in cells under shear stress caveolin-1 is also found in the cytoplasm. Shear stress also increases caveolin-1 concentration in the upstream side of the cells. Our results are also in agreement with a previous study has shown that laminar shear stress increases caveolin-1 expression and it also changes the localization of caveolin-1 [22].

In conclusion, we have introduced a parallel plate flow chamber for shear stress studies in vitro. The data provides robust evidence that this parallel plate flow chamber can be used as an effective in vitro system to study the effects of fluid shear stress on the structure and function of endothelial cells. Advantages of this system include its simplicity of use and small number of cells required to run a simulation. The flow rates can be precisely controlled in this laminar flow chamber to induce shear stress in order to simulate the hemodynamic environment of vasculature in vivo. This flow device provides a leak proof and contamination free environment to the cells. It can also be used to do for real time monitoring of the live cells, to study the dynamics of endothelial cell response to shear. The material used for making this flow device is chemical resistant. The fabrication is convenient, versatile and economical.

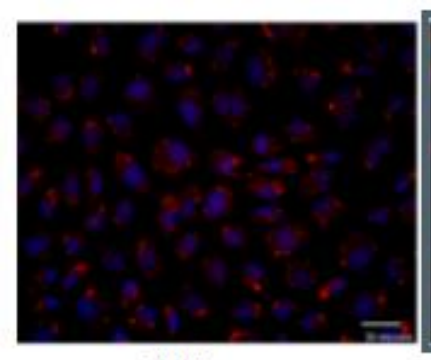

Static

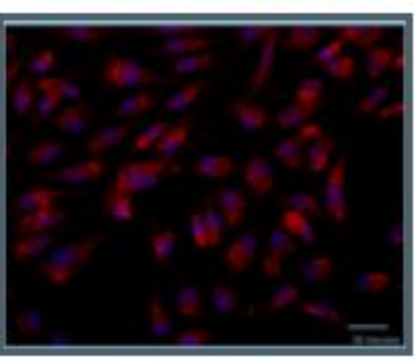

4 dyne $/ \mathrm{cm}^{2}$

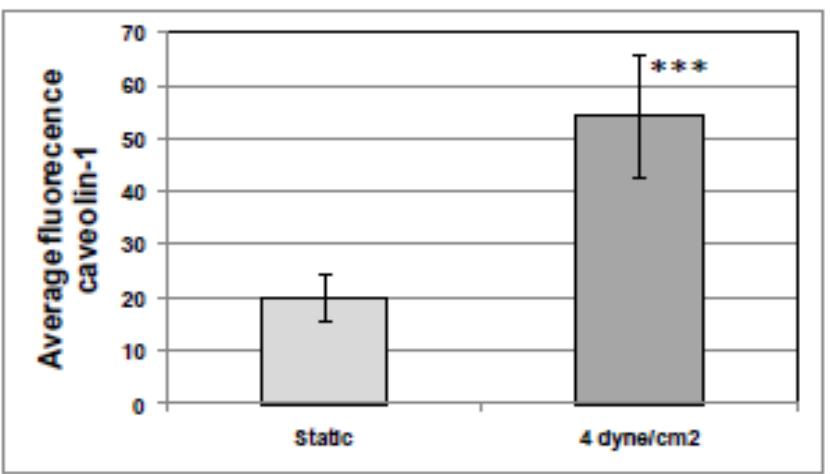

Figure 5. Shear stress upregulates caveolin-1 expression in bovine endothelial cells. (A) BAECs were exposed to shear stress for 16 hours and static cells were used as controls. Caveolin-1 expression and distribution (red) was determined by indirect immunofluorescence. The nuclei were stained with DAPI (blue). The images were taken by $40 \mathrm{X}$ oil emersion objective using Zeiss Axiovert 200 microscope. (B) Average gray values (immunofluorescence intensity) of caveolin-1 levels in cells maintained under static and shear stress. The values (average \pm SD) were calculated from at least 10 different microscopic fields in each slide $(\mathrm{n}=3)$. *** $\mathrm{P}$ $<0.001$.

\section{Acknowledgement}

Supported by Natural Sciences and Engineering Research Council (NSERC) Discovery Grants to B.M. and R.C. 


\section{References}

1. Tricot, O., Mallat, Z.; Heymes, C.; Belmin, J.; Leseche, G.; Tedqui, A. Relation between endothelial cell apoptosis and blood flow direction in human atherosclerotic plaques, Circulation, 2000, 101 (21), 2450 - 2453. doi: 10.1161/01.CIR.101.21.2450

2. Chien, S. Role of shear stress direction in endothelial mechanotransduction, Mol Cell Biomech, 2008, 5(1), 1 - 8.

3. Traub, O.; Berk, B.C. Laminar shear stress: mechanisms by which endothelial cells transduce an atheroprotective force, Arterioscler Thromb Vasc Biol, 1998, 18(5), 677- 685. doi: 10.1161/01.ATV.18.5.677

4. Young, E.W.; Wheeler, A.R.; Simmons, C.A. Matrix-dependent adhesion of vascular and valvular endothelial cells in microfluidic channels, Lab Chip, 2007, 7(12), 1759 - 1766. doi: 10.1039/B712486D

5. Frangos, J.A.; Eskin, S.G.; McIntire, L.V.; Ives, C.L. Flow effects on prostacyclin production by cultured human endothelial cells, Science, 1985, 227(4693), 1477 - 1479. doi: $\underline{10.1126 / \text { science. } 3883488}$

6. Levesque, M.J.; Nerem, R.M. The elongation and orientation of cultured endothelial cells in response to shear stress, J Biomech Eng, 1985, 107(4), 341 - 347.

7. Galbraith, C.G.; Skalak, R.; Chien, S. Shear stress induces spatial reorganization of the endothelial cell cytoskeleton, Cell Motil Cytoskeleton, 1998, 40(4), 317 - 330. doi: 10.1002/(SICI)1097-0169

8. McCann, J.A.; Peterson, S.D.; Plesniak, M.W.; Webster, T.J.; Haberstroh, K.M. Non-uniform flow behavior in a parallel plate flow chamber alters endothelial cell responses, Ann Biomed Eng, 2005, 33(3), 328 - 336. doi: 10.1007/s10439-005-1735-9

9. Bacabac, R.G.; Smit, T.H.; Cowin, S.C.; Van Loon, J.J.; Nieuwstadt, F.T.; Heethaar, R.; Klein-Nuleng, J. Dynamic shear stress in parallel-plate flow chambers, J Biomech, 2005, 38(1),159-167. doi:10.1016/i.jbiomech.2004.03.020

10. Azuma, N.; Akasaka, N.; Kito, H.; Ikeda, M.; Gahtan, V.; Sasajima, T.; Sumpio, B.E. Role of p38 MAP kinase in endothelial cell alignment induced by fluid shear stress, Am J Physiol Heart Circ Physiol, 2001, 280(1), H189 - 197.

11. Wojciak-Stothard, B.; Ridley, A.J. Shear stress-induced endothelial cell polarization is mediated by Rho and Rac but not Cdc42 or PI 3-kinases, J Cell Biol 2003, 161(2), 429 - 439. doi: $10.1083 /$ jcb. 200210135

12. Topper, J.N.; Gimbrone, M.A. Jr.. Blood flow and vascular gene expression: fluid shear stress as a modulator of endothelial phenotype, Mol Med Today, 1999, 5(1), 40 - 46.

13. Wang, J.H.; Goldschmidt-Clermont, P.; Wille, J.; Yin, F.C. Specificity of endothelial cell reorientation in response to cyclic mechanical stretching. J Biomech, 2001, 34(12), 1563 1572.

14. Kadohama, T.; Akasaka, N.; Nishimiura, K.; Hoshino, Y.; Sasajima, T.; Sumpio, B.E. p38 Mitogen-activated protein kinase activation in endothelial cell is implicated in cell alignment and elongation induced by fluid shear stress, Endothelium, 2006, 13(1), 43-50. doi: $\underline{10.1080 / 10623320600660219}$

15. Davies, P.F. Flow-mediated endothelial mechanotransduction, Physiol Rev, 1995, 75(3), 519-60.

16. Kuchan, M.J.; Frangos, J.A. Role of calcium and calmodulin in flow-induced nitric oxide production in endothelial cells, Am J Physiol, 1994, 266(3 Pt 1), C628-36.

17. Andrews, A.M.; Jaron, D.; Buerk, D.G.; Kirby, P.L.; Barbee, K.A. Direct, real-time measurement of shear stress-induced nitric oxide produced from endothelial cells in vitro, Nitric Oxide, 23(4), 335 - 342. doi:10.1016/j.niox.2010.08.003

18. Yang, Z.; Tao, J.; Wang, J.M.; Tu, C.; Xu, M.G.; Wang, Y.; Chen, L.; Luo, C.F.; Tang, A.L.; Ma, H. [Fluid shear stress upregulated endothelial nitric oxide synthase gene expression and nitric oxide formation in human endothelial progenitor cells], Zhonghua Xin Xue Guan Bing Za Zhi, 2007, 35(4): 359 362.

19. Corson, M.A.; James, N.L.; Latta, S.E.; Nerem, R.M.; Berk, B.C.; Harrison, D.G. Phosphorylation of endothelial nitric oxide 
synthase in response to fluid shear stress, Circ Res, 1996, 79(5), 984 - 991. doi: 10.1161/01.RES.79.5.984

20. Dimmeler, S.; Fleming, I.; Fisslthaler, B.; Hermann, C.; Busse, R.; Zeiher, A.M. Activation of nitric oxide synthase in endothelial cells by Akt-dependent phosphorylation, Nature, 1999, 399(6736), 601 - 605. doi:10.1038/21224

21. Smart, E.J.; Ying, Y., Donzell, W.C.; Anderson, R.G. A role for caveolin in transport of cholesterol from endoplasmic reticulum to plasma membrane, J Biol Chem, 1996, 271(46), 29427 - 29435. doi: 10.1074/jbc.271.46.29427

22. Sun, R.J.; Muller, S.; Stoltz, J.F.; Wang, X. Shear stress induces caveolin-1 translocation in cultured endothelial cells, Eur Biophys J, 2002, 30(8), 605 -611. doi: 10.1007/s00249001-0195-X 